\title{
La educación informal en el aprendizaje del español entre los universitarios de Corea del Sur
}

\author{
Jorge Daniel Mendoza Puertas \\ Universidad de Ulsan (Corea del Sur) \\ jormenpue@ulsan.ac.kr
}

Resumen: En la actualidadla educación informal constituye un apoyo fundamental en la adquisición de la competencia comunicativa de cualquier lengua extranjera. En este sentido, este trabajo ofrece un acercamiento a las prácticas informales utilizadas por los estudiantes coreanos de español como lengua extranjera siguiendo una metodología mixta, es decir, cualitativa y cuantitativa. Sobre una muestra de 190 estudiantes, procedentes de tres universidades coreanas, estudiamos la frecuencia con la que realizan este tipo de actividades (leer libros, revistas, periódicos, cómics,...; ver vídeos, programas, películas, series,...; escuchar la radio; escuchar música; hablar con familiares, amigos o conocidos; usar aplicaciones en el móvil y navegar por internet), la importancia que dan a este tipo de aprendizaje, qué tipo de actividad consideran más relevante para el aumento de su competencia en la lengua meta así como las causas por las que no llevan a cabo estas prácticas con más asiduidad. El estudio se apoya en una serie de entrevistas semiestructuradas y en la elaboración de un cuestionario, a partir de la información obtenida en ellas y su contraste con la bibliografía, cuyos datos fueron analizados con un paquete estadístico. Los resultados del estudio revelan que los estudiantes coreanos tienen una percepción positiva sobre la educación informal, si bien no la llevan demasiado a la práctica debido a la falta de tiempo y a un desconocimiento general sobre el acceso a las fuentes. También se constató 
que el nivel de lengua influye, por un lado, en la percepción del estudiante sobre este tipo de educación y, por otro, en la frecuencia de realización de estas prácticas.

Palabras clave: educación informal, ELE, Corea del Sur, universidad.

\title{
Informal Education in Learning Spanish among South Korean University Students
}

\begin{abstract}
Nowadays it is well-known that informal learning is an important support in acquiring communicative competence in any target language. In this sense, this paper offers an approach to the informal education practices carried out by Korean students of Spanish as a Foreign Language. The research was conducted following a mixed methodology: qualitative and quantitative. On a sample of 190 learners from three Korean universities, we studied the frequency of these practices, the grade of importance they gave to these tasks and which activity was considered the most important for developing their competence. Additionally, we look at the reasons why they do not carry out these practices more often. The results revealed that although Korean learners have a positive perception of informal education, they do not spend too much time on these kinds of activities, mainly due to their lack of time and their ignorance of the many resources accessible to them. Moreover, the research showed that the students' level of Spanish has an influence over their perception of this kind of learning and over the frequency they carry out these informal tasks.
\end{abstract}

Keywords: informal education, SFL, South Korea, university.

Recibido el 15/04/2018

Aceptado el 09/10/2018 


\section{Introducción}

El aprendizaje de cualquier lengua extranjera suele ser un camino largo y complejo. La lentitud es uno de los rasgos más característicos de este proceso, además de estar lleno de vaivenes, de momentos de avance y retroceso, y de encontrarse condicionado por multitud de factores tanto individuales como sociales (Palacios, 2006). En un recorrido de tales características, la acción desarrollada en el aula resulta por sí misma insuficiente. Las tareas abordadas en clase así como las estrategias y contenidos allí aprendidos deberían constituir el punto de inicio que, a modo de trampolín, sirviera al aprendiente para entrar en contacto con la lengua meta en contextos más reales y menos limitados. En esta línea, Palacios (2006) considera que las pautas y herramientas suministradas en el aula deben servir al alumno para aprender por sí solo fuera de ella sin la presencia del profesor. Igualmente, Arslan (2011) afirma que las lenguas extranjeras comienzan a adquirirse en la escuela, pero se practican fuera de ella. Por lo que las actividades de clase resultan tan importantes como las que se desarrollan más allá de las paredes de esta. El contacto frecuente con la lengua objeto no solo facilita la asimilación y fijación de los contenidos estudiados sino que propicia la adquisición de otros múltiples elementos léxicos, pragmáticos, sintácticos, etc. garantizando el avance en la adquisición de la misma. Según Corpas (2016, p. 76), este tipo de educación «proporciona excelentes oportunidades para adquirir, afianzar y consolidar las destrezas propias de las lenguas». Pero todo esto exige, por parte del aprendiente, tanto el desarrollo de una cierta autonomía como una implicación en el propio proceso de aprendizaje que vaya más allá de los entornos tradicionales de enseñanza. Por tanto, el estudiante debería complementar su formación oficial con una serie de actividades extracurriculares. En este sentido, no podemos olvidar que en la actual sociedad del conocimiento se ha desplazado el centro de gravedad en la educación; de modo que «el profesorado hace de tutor, de guía, pero ha de ser el propio estudiante el que aprenda, el que consiga autonomía y el que tenga necesidad de tomar iniciativas para llevar a cabo su aprendizaje» (Romea, 2011, p. 112). Todo esto favorecido por las numerosas herramientas tecnológicas así como por el fácil acceso a la información y a la comunicación.

El desarrollo de la competencia comunicativa en cualquier lengua pasa, sin duda, por este tipo de aprendizaje informal del que hablamos, muy especialmente en aquellos entornos donde la exposición a la lengua meta y el contacto natural con la misma es tan extraño como difícil. Una situación a la que se enfrenta la mayoría de los aprendientes de español en un país como Corea del Sur ${ }^{1}$. A esto

1. Esta afirmación parte de la propia experiencia del autor tras 5 años como docente universitario en Corea del Sur. También puede consultarse al respecto Miró y Álvarez (2015). 
se le une, como expondremos a lo largo de este texto, la dificultad de acceso, de buena parte de este colectivo, a la llamada educación no formal. De este modo, teniendo en cuenta el peso que adquiere la educación informal en nuestro contexto para una adecuada adquisición del español, esta investigación pretende ofrecer un acercamiento a las actividades de carácter informal que realizan los estudiantes universitarios de ELE. Más concretamente, con este trabajo pretendemos conocer:

- Qué actividades informales desarrollan los estudiantes universitarios de ELE y con qué frecuencia.

- Qué actividades consideran más útiles.

- Si estos son conscientes del valor de la educación informal en su proceso de aprendizaje del español.

- Si existe alguna relación entre la percepción de este tipo de aprendizaje y el nivel de español.

- Si existe relación entre la frecuencia de realización de estas actividades y su nivel de español.

- Las razones por las que no dedican más tiempo a estas actividades.

\section{Educación informal y aprendizaje de lenguas extranjeras}

El sintagma educación informal, lejos de ser un concepto fácil de definir, puede ser entendido e interpretado de maneras diversas. Como Tudor (2013) indica, no hay una definición completamente respaldada por los teóricos. Autores como Doyle (2001), Mahoney (2001), Wolfe y Richardson (2001) o Smith (2001) consideran la educación informal como aquella que se lleva a cabo fuera de las instituciones oficiales de enseñanza, en entornos diversos, por parte de educadores que suelen trabajar con comunidades o asociaciones y que promueven un tipo de aprendizaje centrado más en el proceso que en los resultados. También hay quienes entienden la educación informal como un estilo de enseñanza-aprendizaje. Este se situaría dentro de un continuum cuyo extremo opuesto sería la enseñanza formal, de modo que cualquier profesor o educador podría ir moviéndose en sus sesiones dentro del mismo según su modo de enseñanza se aproximara más al concepto de aprendizaje como resultado o como proceso (Ellis, 1990; Mahoney, 2001). Para otros, como Livingstone (2001), el aprendizaje informal abarca cual- 
quier actividad que involucre la búsqueda de la comprensión, el conocimiento o el dominio de una habilidad, y que ocurre sin la existencia de criterios curriculares externos. Scribner y Cole (1973) nos hablan de aquella educación de carácter continuo y completamente contextualizada que se produce en nuestra vida diaria, generalmente a través de la observación, en la que los aspectos prácticos, tradicionales y emocionales tienen mucha más importancia que en la educación formal. Por otro lado, atendiendo a la cuestión terminológica, ciertos investigadores prefieren nombrar este concepto utilizando el sustantivo aprendizaje y no educación, por cuanto en su desarrollo no existen instituciones educativas o profesores autorizados implicados, e igualmente no hay un plan de estudios prescrito (Schugurensky, 2000). Sin embargo, ambas etiquetas, educación informal y aprendizaje informal, suelen utilizarse como sinónimos la mayoría de las veces.

Como hemos pretendido exponer en este somero esbozo, la delimitación del tema abordado es compleja y amplia. No obstante, quizás la forma más sencilla de aproximarse al concepto de educación informal es definirlo por contraste con los otros tipos de educación existentes. A este respecto, las definiciones ofrecidas por Coombs, Prosser y Ahmed (1973) resultan esenciales:

- Educación formal: Es el sistema educativo jerárquicamente estructurado y cronológicamente calificado, que va desde la escuela primaria hasta la universidad e incluye, además de estudios académicos generales, una variedad de programas especializados e instituciones para la formación profesional y técnica.

- Educación no formal: Se refiere a cualquier actividad educativa organizada fuera del sistema formal, cuya finalidad es servir a clientes de aprendizaje y cuyos objetivos de aprendizaje son identificables.

- Educación informal: Se relaciona con el aprendizaje a lo largo de la vida, en el que cada individuo adquiere actitudes, valores, habilidades y conocimientos de la experiencia diaria y las influencias y recursos educativos en su entorno.

Como es posible apreciar, la definición de educación informal parece aludir a un conocimiento de adquisición libre y espontánea directamente relacionado con las necesidades e intereses de cada individuo a lo largo de su vida (Farmahini, Hasan y Noroozi, 2014). 
En un acercamiento más preciso a la noción de educación informal en la actualidad, Cuadrado (2008) ofrece un estudio bastante completo de sus rasgos. Sintetizamos algunas de sus líneas definitorias:

- Características: Concepción lúdica y no organizada, no necesita de la intervención de un profesor, su acción no es visible socialmente, el nivel de consciencia de los conocimientos adquiridos es muy bajo, dura toda la vida, es más atractiva, entretenida y divertida, el individuo desencadena el proceso de aprendizaje, etc.

- Contenidos: Abarca un amplio espectro, no son contenidos explícitos como en la educación formal y no formal, sus contenidos son la educación para la vida misma.

- Campos de actuación: Son innumerables, por ejemplo, el amor, el arte, la calle, la enfermedad, el folclore, el juego, la fiestas, la moda, etc.

+ Espacios: «(...) espacios de convivencia donde se producen relaciones sociales, el contacto con la realidad, los productos culturales o las acciones comunicativas sin mediación pedagógica» (p. 54).

- Agentes: Son muy numerosos, a saber, la familia, los amigos, la música, los cómics, la prensa, la televisión, la publicidad, los juguetes, los refranes, la vida social, etc.

Otra de las cuestiones muy debatidas en torno a la educación informal ha sido su carácter intencional. Trilla (1986) habla de la no intencionalidad como una de las características distinguidoras de este tipo de educación, aunque, a su vez, se cuestiona si toda la educación informal puede considerarse no intencional. Discrepa en este punto Quintana (1991), quien afirma que no puede hablarse de educación informal si esta implica ausencia de intención, por cuanto el acto educativo exige que un educador se ponga a educar y eso requiere intencionalidad. Por su parte, Touriñán (1996) considera que a los procesos informales se les llama así porque obtienen resultados con valor educativo por medio de estímulos no directamente educativos, pero no niegan el carácter intencional de la educación. En relación con este tema, Shugurensky (2000) consideró necesario desarrollar una taxonomía de formas de aprendizaje informal basándose en esta categoría esencial, la intencionalidad, a la que añade una segunda, la conciencia. De este modo, diferencia tres tipos de aprendizaje: 
- Autónomo.- Se refiere a proyectos de aprendizaje llevados a cabo por individuos, sin el apoyo de un educador, de manera intencional y consciente.

- Incidental. - Es involuntario, pero consciente. El individuo no tiene ninguna intención de aprender algo de una determinada experiencia, pero una vez concluida se da cuenta de que se ha producido un aprendizaje.

+ Socialización. - Consiste en la internalización de valores, actitudes, habilidades, comportamientos, etc. que ocurren durante la vida cotidiana. El sujeto no tiene intención de adquirirlos y tampoco es consciente de lo que ha aprendido.

De acuerdo con esta clasificación, el aprendizaje informal que pueden llevar a cabo nuestros estudiantes de ELE sería de carácter autónomo. Puesto que en un contexto de no inmersión, y tan ajeno a la lengua española como es Corea del Sur, cualquier contacto establecido con la lengua meta fuera del aula es buscado (intencional) y con una conciencia de aprendizaje vinculado a este.

Una vez descritos los conceptos básicos, expondremos algunas actividades propias del aprendizaje informal que podrían llevar a cabo nuestros estudiantes ${ }^{2}$ :

Ver vídeos, series, programas, documentales, películas, etc. - Aun cuando en Corea no existen programas en español en la TV, el actual acceso a internet permite a cualquier estudiante entrar en contacto con los materiales audiovisuales anteriormente expuestos. Existen páginas de diferentes canales de televisión de países hispanos donde pueden acceder a contenidos, pero también es posible encontrar vídeos de distinto tipo (desde series, pasando por programas, noticias o documentales) en plataformas como Youtube, Vimeo, Dailymotion, etc. También es posible encontrar este material para distintos niveles, por ejemplo, ciertos dibujos animados para niños son idóneos para niveles iniciales. Por otro lado, las bibliotecas universitarias suelen tener películas en español que podrían ver haciendo uso del servicio de préstamo.

Leer libros, revistas, periódicos, cómics, etc. - En Corea tampoco es fácil encontrar libros y mucho menos revistas o periódicos en español. Es raro localizar en las librerías internacionales alguna sección en nuestra lengua que, si existe, suele estar dedicada a libros de ELE escritos en coreano. No obstante, aquí pueden servirnos tanto los libros y recursos que tenga la biblioteca universitaria como aquellos existentes en internet, nuestra gran aliada. Debido a la actual globalización, algunos estudiantes son seguidores de diversos personajes públicos españoles o latinoamericanos del mundo del deporte, del cine, de la música,...

2. Para la enumeración de actividades, partimos de Corpas (2016) y añadimos algunas propuestas basadas en nuestra experiencia docente en la República de Corea. 
Buscar información en español acerca de estos, en revistas o periódicos en línea, puede ser una buena oportunidad para desarrollar su comprensión escrita. Otra opción interesante y lúdica son las tiras cómicas de diversos ilustradores famosos, ciertos cuentos y cómics. Del mismo modo, muchos de ellos han estudiado literatura española y latinoamericana, y poseen autores favoritos. Acercarse a algunos fragmentos de sus obras, desde el más puro placer o interés literario, constituye otra posibilidad interesante.

Escuchar la radio.- Esta actividad, que puede realizarse de forma pasiva mientras hacemos otras tareas, permite entrar en contacto con numerosos temas y contenidos. Obviamente, internet facilita el acceso a emisoras de diversos países. Es ideal para estudiantes que han pasado algún tiempo fuera de Corea y tienen interés en seguir la actualidad del país donde han estado ${ }^{3}$.

Escuchar música.- Desde hace unos años, la música hispanoamericana y española ha empezado a escucharse en muchos lugares del mundo. Incluso en Corea, no es extraño escuchar, de vez en cuando, alguna canción de moda en español. Nuestros estudiantes suelen conocer algunos cantantes latinos, hecho que aumenta tras su estancia por estudios en el extranjero. De este modo, escuchar canciones es una manera divertida de entrar en contacto con la lengua meta e ir aprendiendo cosas nuevas de forma natural.

Navegar por internet.- Aunque hoy en día es poco probable, todas las actividades hasta aquí expuestas podrían realizarse sin internet. El mero hecho de navegar por la red, en momentos de ocio, podría considerarse una actividad en sí misma. Esta acción no organizada, de búsqueda de información diversa o de carácter lúdico, nos hace entrar en contacto con numerosos contenidos que enriquecen sin duda la lengua objeto de estudio.

Hablar con amigos, familiares o conocidos.- Practicar la conversación implica grandes beneficios para el aprendizaje de una lengua extranjera. Más allá del input recibido, la producción oral lleva asociadas importantes ventajas. De este modo, no solo mejora la fluidez (Arnaiz y Peñate, 2004), sino, como ya apuntaron tanto Swain $(1985,1995)$ como Swain y Lapkin (1995), facilita la toma de conciencia sobre lagunas lingüísticas existentes en el discurso, contribuye a la comprobación de hipótesis, conduce del procesamiento semántico al sintáctico y hace reflexionar sobre la propia lengua.

Algunos estudiantes han vivido en Hispanoamérica y tienen algún familiar que también puede hablar español, mientras que otros han disfrutado de una estancia por estudios en el extranjero y tienen conocidos o amigos en otros países. En ocasiones, también pueden encontrar algún estudiante de intercambio en la

3. Interés constatado no solo a través de la experiencia personal del autor sino también mediante las entrevistas realizadas para este y otros trabajos. 
universidad que sea hablante nativo de español. Conversar presencialmente o a través de un servicio de videollamada se convierte en una gran oportunidad para mejorar sus habilidades comunicativas y aprender de manera informal.

Uso de aplicaciones en teléfonos móviles. - La idoneidad de los móviles y las tabletas como herramientas para el aprendizaje autónomo de lenguas extranjeras ha sido constatada por investigadores como Chen (2013), Clough, Jones, McAndrews y Scalon (2009), y Godwin-Jones (2011), entre otros. No en vano, estos dispositivos ofrecen una multitud de empleos debido a su conectividad a internet así como a las muy diversas aplicaciones para el estudio de lenguas que han ido surgiendo en estos últimos años.

\section{La educación en la República de Corea más allá del sistema formal}

La sociedad de Corea del Sur concede una importancia notoria a la educación (Jiménez y Cabrera, 2011). Esta constituyó, en el pasado siglo, una de las piedras angulares del milagro económico nacional ${ }^{4}$. La educación corría en paralelo a los planes para el desarrollo económico del país, que consiguieron sacar a Corea de la más absoluta pobreza y convertirla en una de las economías mundiales más potentes.

En la actualidad, la mayoría de los coreanos está convencida de que el camino hacia una carrera y vida exitosas se basa en una buena educación (Bermeo, 2014; Lee, 2006; Onieva, 2015; Seth, 2002). Un convencimiento que encuentra sus orígenes en los valores confucianos tan arraigados en la nación (Lee, 2006; Onieva, 2015). La tradición educativa confuciana se concretó en el periodo Joseon (1392-1910) en un sistema meritocrático basado en la superación de exámenes para acceder a los puestos de poder y cultura; aunque los yangban, o clase gobernante, terminarían monopolizando la educación superior para garantizar el mantenimiento del estatus socio-político propio y de sus clanes familiares (Lee, 2006). Con la democratización de la educación tras el periodo de dominación japonesa, se pierde el clasismo imperante en el sistema histórico pero su fuerte impronta meritocrática se mantiene. No en vano, como apunta Lee (2006), los antiguos exámenes estatales para reclutar funcionarios públicos (kwa-keo) son considerados la base del actual sistema de oposiciones para formar parte del cuerpo de funcionarios del estado, así como la base del famoso sistema de exámenes de acceso a la universidad (suneung). Aludiendo a este último sistema, la exigencia de las pruebas es algo sobradamente conocido (Ellinger y Carlson, 1990; Onieva,

4. De la Cruz (2014) nos recuerda que las políticas de I+D y educación fueron centrales en el modelo exitoso de los llamados Estados Desarrollistas del nordeste asiático (Japón, Corea del Sur y Taiwán). 
2015). Estos exámenes constituyen uno de los momentos más decisivos en la vida de cualquier estudiante. Entrar en una reconocida institución puede marcar la diferencia entre un futuro prometedor y un futuro incierto ${ }^{5}$. De este modo, conseguir ser alumno de la prestigiosa Universidad Nacional de Seúl se sigue considerando un privilegio y un orgullo para la familia. Como puede intuirse, la competición por superar este riguroso proceso de selección es extrema (Shin y Koh, 2005). Todo esto conlleva una evidente presión formativa bajo la que vive la población joven ${ }^{6} y$ cuyas consecuencias han servido para definir una de las características más singulares de la realidad educativa coreana contemporánea, nos referimos a la gran oferta y demanda de educación no formal. Ante una sociedad que mide la capacidad personal por el número y la filiación de los diplomas que ostenta un individuo ${ }^{7}$, la inversión en educación privada y la dependencia de ella son grandes ${ }^{8}$. Así lo denunciaba Ahn (2012, p. 1) hace algunos años: «Parents also believe they cannot rely on public education alone to get their children into the desired colleges, leading to an enormous dependence on private education».

Según indican Hultberg et al. (2017), el gasto actual medio de una familia en educación no formal es de unos 244000 wones, 217 dólares estadounidenses, por mes e hijo. Muy diversas academias o hagwons proliferan por todo el país compitiendo por captar estudiantes. En este sentido, las academias de inglés son numerosísimas. Tras el acceso a la universidad la demanda de educación no formal no termina, ya que hay que obtener diferentes certificados de idiomas, informática y demás, que se consideran indispensables en cualquier currículum. Si, como hemos dicho, la presencia de academias de inglés es avasalladora, los hagwons de español no corren la misma suerte. De hecho, si salimos de Seúl, tan solo es posible encontrar algunos en Busan, la segunda ciudad más importante del país. Esta realidad entra en contradicción con el creciente interés por nuestro idioma que existe por tierras coreanas ${ }^{9}$. De este modo, nuestros estudiantes, así como todos aquellos alumnos de otros departamentos de español ubicados fuera de estas

\footnotetext{
5. Estudiar en una de las tres mejores universidades del país, el llamado grupo SKY (Universidad Nacional de Seúl, Universidad de Corea, Universidad Yonsei), asegura una valiosa red de contactos y casi garantiza conseguir trabajo en el gobierno o en uno de los 63 chaebols, como Samsung, Lotte, Hyundai o LG (Hultberg et al., 2017).

6. Existe una alta tasa de suicidio entre jóvenes vinculada a esta causa (Lee, Hong y Espelage, 2010).

7. Lee (2006) denuncia el influjo que ejercen los logros en educación superior y las redes de contactos a la hora de encontrar trabajo, frente a las verdaderas habilidades y aptitudes de un individuo. Destacando que aquellos que carecen de títulos universitarios no tienen nada fácil acceder al empleo.

8. Dos críticas se asocian a este hecho: por un lado, el aumento de la brecha social entre ricos y pobres, y, por otro, el cuestionamiento sobre la calidad de la educación pública nacional (Ahn, 2012; Hultberg et al., 2017; Lee, 2006).

9. Para saber más sobre los datos relacionados con el estudio del español en la actualidad en Corea del Sur, véase Hernández (2015). También pueden consultarse Jiménez y Cabrera (2011), y Miró y Álvarez (2015).
} 
dos ciudades, no tienen acceso a la educación no formal. La única alternativa es contratar a un profesor particular nativo (que no son nada fáciles de encontrar fuera de la capital) cuyos honorarios, de acuerdo con el precio medio de las clases particulares en Corea, oscilan entre los 50 y 80 dólares la hora. Una opción que no muchos pueden permitirse. Es por ello que el desarrollo de la competencia en español de un gran número de estudiantes coreanos solo encuentra apoyo en un aprendizaje autónomo dentro de lo que hemos denominado educación informal.

\section{Procedimiento}

Este estudio se ha llevado a cabo sobre una muestra de 190 estudiantes de español procedentes de tres universidades coreanas: la Universidad de Ulsan, la Universidad Católica de Daegu y la Universidad Nacional de Seúl. El número de encuestados vinculado a cada una de las instituciones es diverso: Universidad de Ulsan (116), Católica de Daegu (68), Nacional de Seúl (6). Los informantes de esta última eran de posgrado, mientras que aquellos de las dos primeras eran de los cuatro cursos que componen el grado. La distribución por cursos es la siguiente: $65\left(1 \circ^{\circ}\right), 45\left(2 .^{\circ}\right), 40\left(3 .^{\circ}\right), 34\left(4 .^{\circ}\right), 6$ (máster $)^{10}$. De ellos 56 eran hombres y 134 mujeres $^{11}$; 53 habían estudiado español en el extranjero y 137, no ${ }^{12}$.

El procedimiento seguido se compone de dos fases: una primera fase de entrevistas y una segunda basada en la distribución y análisis de un cuestionario. En la fase de entrevistas participaron un total de 5 estudiantes $^{13}$, uno por cada curso participante en este estudio. Las entrevistas eran semiestructuradas y oscilaron entre los 15 y los 30 minutos de duración. En ellas se realizó una serie de preguntas abiertas, relacionadas con los principales objetivos de este estudio, de las que surgieron otras cuestiones no planificadas según el discurso de cada informante. Las mencionadas entrevistas fueron grabadas, transcritas, analizadas y contrastadas con la información procedente de la bibliografía con el fin de crear nuestro cuestionario. El cuestionario utilizado se encuentra formado por tres partes: la primera, destinada a recabar los datos personales de los informantes; la segunda,

10. La diferenciación por cursos no resulta una variable de interés para el presente trabajo (véase nota 12). En las líneas previas nos limitamos a describir la muestra.

11. La diferenciación por sexo no es tomada como variable para este trabajo, pero queremos dejar constancia de la presencia de hombres y mujeres en la descripción de la muestra.

12. Para medir el nivel de español, utilizamos como variable la estancia en el extranjero. Una vez terminado el primer curso, la posibilidad de elegir asignaturas e inscribirse en ellas sin un filtro real de nivel no hace que el aumento de curso implique una mejora necesaria en la competencia en español; sin embargo, la estancia por estudios en algún país hispano, algo opcional, conlleva una diferencia cualitativa a este respecto.

13. Según Kvale (2011), cinco suele ser el número mínimo de informantes en los estudios más comunes basados en entrevistas. Un número más que suficiente para un estudio mixto o combinado como este (Pole, 2009). 
formada por una escala de Likert de 7 ítems ${ }^{14}$ y 5 puntos ${ }^{15}$, destinada a sondear en qué grado realizan distintas actividades nuestros encuestados; la tercera, constituida por una serie de cuestiones de respuesta dicotómica, politómica o abierta, cuya finalidad es aproximarse a la percepción que tienen los estudiantes sobre este tipo de aprendizaje y las causas de su posible escasa práctica ${ }^{16}$. Cabe destacar que la escala tipo Likert ofreció una alta fiabilidad al presentar un coeficiente alfa de Cronbach de 0,840. Los cuestionarios fueron distribuidos durante el segundo semestre del curso 2017 y sus datos fueron analizados con el paquete SPSS22 para Windows. En el análisis de los datos se emplearon frecuencias, porcentajes, estadísticos descriptivos básicos, tablas de contingencia, y pruebas no paramétricas: la prueba U de Mann-Whitney, para la comparación de variables ordinales, así como la ji al cuadrado de Pearson y la prueba exacta de Fisher, para determinar la dependencia o independencia de variables de tipo nominal o de tipo nominal y ordinal.

\section{Resultados}

\subsection{Actividades informales y frecuencia de realización}

Atendamos, en primer lugar, a los resultados de la escala de Likert, con la que nos proponíamos conocer en qué grado llevaban a cabo distintas actividades informales los estudiantes coreanos de ELE. Si echamos una ojeada a los datos de los estadísticos descriptivos, podremos empezar a formarnos una idea sobre los resultados generales ${ }^{17}$ :

\begin{tabular}{|r|r|r|r|r|r|r|r|}
\cline { 2 - 8 } \multicolumn{1}{c|}{} & Leer & Vídeos & Radio & Música & Hablar & Móvil & Internet \\
\hline Media & 2,00 & 2,09 & 1,47 & 2,74 & 2,19 & 2,65 & 3,01 \\
\hline Mediana & 2,00 & 2,00 & 1,00 & 3,00 & 2,00 & 3,00 & 3,00 \\
\hline Moda & 1 & 1 & 1 & 3 & 1 & 1 & 3 \\
\hline
\end{tabular}

Centrémonos, en primer lugar, en las medidas de tendencia central. Fijándonos en la media, si tenemos en cuenta que el máximo de cada ítem por informante sería 5, y observamos que la mayoría de ellos no alcanza los dos puntos y medio

14. Se pregunta la frecuencia con la que realizan las siguientes actividades en español: leer libros, revistas, periódicos, cómics,...; ver vídeos, programas, películas, series,...; escuchar la radio; escuchar música; hablar con familiares, amigos o conocidos; usar aplicaciones en el móvil y navegar por internet.

15. Donde el 1 sería nunca y el 5, muy frecuentemente.

16. Las respuestas dadas en la fase de entrevistas nos llevó a considerar que este último apartado podría ser planteado como objetivo y, por tanto, incluido en nuestro cuestionario.

17.En las tablas utilizaremos una sola palabra para identificar cada ítem. Para más información véase la nota 14. 
o bien lo supera levemente, podremos imaginar que existe un mayor número de respuestas de baja puntuación. Como vemos, la mediana, que indica el lugar central entre todos los valores del conjunto de datos, muestra valores muy próximos a las medias. Especialmente interesante en la interpretación de este tipo de escala es la moda. Esta medida revela el dato que más veces se repite, es decir, aquel con mayor frecuencia absoluta. Las modas de nuestros ítems confirman la tendencia a una baja puntuación por parte de la mayoría de los encuestados. A este respecto, excepto en escuchar música y navegar por internet, la respuesta que más se repite es la de valor uno, o sea, la respuesta nunca.

A continuación, ofrecemos una tabla detallada con las frecuencias (f) y porcentajes (\%) obtenidos por cada actividad en cada uno de los puntos de la escala ${ }^{18}$;

\begin{tabular}{|c|c|c|c|c|c|c|c|c|c|c|c|c|c|c|}
\hline & \multicolumn{2}{|c|}{ Leer } & \multicolumn{2}{|c|}{ Vídeos } & \multicolumn{2}{|c|}{ Radio } & \multicolumn{2}{|c|}{ Música } & \multicolumn{2}{|c|}{ Hablar } & \multicolumn{2}{|c|}{ Móvil } & \multicolumn{2}{|c|}{ Internet } \\
\hline & $\mathrm{f}$ & $\%$ & $\mathrm{f}$ & $\%$ & $\mathrm{f}$ & $\%$ & $\mathrm{f}$ & $\%$ & $\mathrm{f}$ & $\%$ & $\mathrm{f}$ & $\%$ & $\mathrm{f}$ & $\%$ \\
\hline $\mathrm{N}$ & 73 & 38,4 & 65 & 34,2 & 130 & 68,4 & 40 & 21,1 & 66 & 34,7 & 57 & 30,0 & 38 & 20,0 \\
\hline $\mathrm{CN}$ & 58 & 30,5 & 54 & 28,4 & 39 & 20,5 & 30 & 15,8 & 58 & 30,5 & 28 & 14,7 & 25 & 13,2 \\
\hline $\mathrm{AV}$ & 46 & 24,2 & 60 & 31,6 & 14 & 7,4 & 70 & 36,8 & 39 & 20,5 & 47 & 24,7 & 54 & 28,4 \\
\hline $\mathrm{F}$ & 12 & 6,3 & 10 & 5,3 & 6 & 3,2 & 39 & 20,5 & 18 & 9,5 & 41 & 21,6 & 44 & 23,2 \\
\hline MF & 1 & 0,5 & 1 & 0,5 & 1 & 0,5 & 11 & 5,8 & 9 & 4,7 & 17 & 8,9 & 29 & 15,3 \\
\hline Total & 190 & 100 & 190 & 100 & 190 & 100 & 190 & 100 & 190 & 100 & 190 & 100 & 190 & 100 \\
\hline
\end{tabular}

Como puede observarse en la tabla, cinco de las siete actividades consultadas presentan en la respuesta nunca su mayor frecuencia y porcentaje en comparación con el resto de las respuestas de la encuesta: leer (73/38,4\%), vídeos (65/34,2\%), radio $(130 / 68,4 \%)$, hablar $(66 / 34,7 \%)$ y móvil (57/30\%). Destaca por encima de todas la actividad de escuchar la radio, donde el casi el $70 \%$ de los informantes respondieron nunca. El resto de los ítems, tras sumar los porcentajes de las respuestas nunca y casi nunca, alcanzan valores comprendidos entre el $60 \%$ y $70 \%$ del total: leer $(131 / 68,9 \%)$, vídeos (119/62,6\%), hablar $(124 / 65,2 \%)$, a excepción del uso de aplicaciones en el móvil, donde la suma de los dos puntos negativos no llega al 45\% (85/44,7\%). En efecto, en esta última un mayor número de estudiantes respondieron positivamente, así, la suma de los dos puntos positivos (frecuentemente y muy frecuentemente) supera el 30\% (58/30,5\%), frente a los valores sensiblemente inferiores de los otros ítems mencionados: leer (13/6,8\%), vídeos

18. Para ahorrar espacio usamos iniciales en los grados de la escala: nunca $(\mathrm{N})$, casi nunca (CS), a veces (AV), frecuentemente (F), muy frecuentemente (MF). 
$(11 / 5,8 \%)$, radio $(7 / 3,7 \%)$, y hablar $(27 / 14,2 \%)$. Ciñéndonos exclusivamente a las respuestas positivas de la escala y dejando a un lado aquella que podría considerarse neutra (a veces), el uso de aplicaciones en el móvil se sitúa entre las actividades informales realizadas con mayor frecuencia. Junto a esta encontramos escuchar música $(50 / 26,3 \%)$ y navegar por internet $(73 / 38,5 \%)$, cuyas respuestas positivas se ubican por encima del $25 \%$ del total. De este modo, navegar por internet sería la actividad informal realizada más frecuentemente por un mayor número de aprendientes $(38,5 \%)$, seguida de usar aplicaciones en el teléfono móvil $(30,5 \%)$ y de escuchar música (26,3\%). No obstante, el número de informantes que apenas realiza estas mismas actividades se acerca bastante o incluso supera las cifras positivas: internet (63/33,2\%), móvil (85/44,7\%), música (70/36,9\%). En consecuencia, podemos afirmar que la muestra analizada no realiza con demasiada frecuencia tareas informales en su aprendizaje del español.

Para saber si existe alguna relación significativa entre el nivel de lengua y la frecuencia de realización de estas actividades, sometimos estas variables y la variable estancia en el extranjero a la prueba U de Mann-Whitney. En todas ellas, se obtuvo un valor p cercano a cero, excepto en la variable móvil, donde el valor fue de 0,067 ( $p>0,05$ ). Estos resultados ponen de manifiesto que existen diferencias en la frecuencia de realización de todas las actividades en función del nivel de español, excepto en el uso de aplicaciones en el móvil. Es decir, aquellos que tienen un mayor nivel tienden a realizar con más frecuencia todas las prácticas señaladas.

\subsection{Percepción de utilidad}

El siguiente punto de nuestro cuestionario sondeaba la percepción que los encuestados tenían sobre la utilidad de este tipo de tareas en su aprendizaje del español. A este respecto, 179 respondieron afirmativamente, considerando este tipo de actividades informales como algo útil para la mejora de su español. Tan solo 11 estudiantes respondieron negativamente. Teniendo en cuenta estos datos, nos cuestionamos si es posible establecer alguna relación significativa entre el periodo de estudios en el extranjero y, por tanto, el nivel de lengua, y la idea que poseen los informantes sobre este tipo de aprendizaje. La siguiente tabla de contingencia puede arrojar alguna luz al respecto:

\begin{tabular}{|c|c|c|c|c|}
\hline & \multicolumn{2}{|c|}{ Utilidad } & \multirow[b]{2}{*}{ Total } \\
\hline & & Sí & No & \\
\hline \multirow{2}{*}{$\begin{array}{l}\text { Estancia por estudios } \\
\text { en el extranjero }\end{array}$} & No & 126 & 11 & 137 \\
\hline & Sí & 53 & 0 & 53 \\
\hline & Total & 179 & 11 & 190 \\
\hline
\end{tabular}


Como puede apreciarse, los 11 encuestados que no consideraron útiles estas actividades solo habían estudiado español en Corea. Por el contrario, todos los estudiantes que habían estudiado fuera tienen una visión positiva sobre este tipo de prácticas. Para conocer si existe algún tipo de relación entre estas dos variables las sometimos a la prueba de la ji al cuadrado, que arrojó un valor p de 0,034. Sin embargo, dado que más del $20 \%$ de las casillas tuvo un recuento menor que 5 , realizamos otra prueba de carácter exacto como el test de Fisher, el cual reveló un valor $\mathrm{p}$ de 0,036 ( $\mathrm{p}<0,05$ ). Rechazamos, pues, la hipótesis nula y constatamos que existe asociación entre ambas variables.

Por otro lado, dejamos una cuestión abierta para que expusieran sus razones acerca de la respuesta elegida. Así, los estudiantes que consideraron útiles este tipo de tareas destacaron una serie de puntos positivos vinculados a estas: su variedad, su flexibilidad, su carácter lúdico, la posibilidad de elección de acuerdo a los intereses personales, la ausencia de estrés en su práctica al realizarse fuera de clase, etc. Y como efectos resultantes aludieron a la mejora que producían en sus destrezas lingüísticas, en la asimilación de los contenidos vistos en el aula y en el aprendizaje de expresiones coloquiales contextualizadas. Aquellos que negaron su utilidad definieron estas actividades como algo difícil de hacer e incidieron en la facilidad con que olvidaban lo aprendido, además de cuestionar su validez por quedar fuera del sistema formal de enseñanza.

\subsection{Mejor actividad informal}

Otro punto de nuestro cuestionario consistía en que señalaran qué actividad, de las expuestas en la escala de Likert, consideraban mejor para el aprendizaje informal del español. Veamos las respuestas en la siguiente tabla:

\begin{tabular}{|c|c|c|}
\hline & \multicolumn{2}{|c|}{$\begin{array}{l}\text { Mejor actividad } \\
\text { informal }\end{array}$} \\
\hline & $\mathrm{f}$ & $\%$ \\
\hline Leer & 9 & 4,7 \\
\hline Vídeos & 63 & 33,2 \\
\hline Radio & 2 & 1,1 \\
\hline Música & 15 & 7,9 \\
\hline Hablar & 91 & 47,9 \\
\hline Móvil & 6 & 3,2 \\
\hline Internet & 4 & 2,1 \\
\hline Total & 190 & 100,0 \\
\hline
\end{tabular}


Como muestran los datos, las actividades mejor valoradas fueron bablar $(91 / 47,9 \%)$ y ver vídeos, programas, etc. $(63 / 33,2 \%)$. Hablar es con diferencia la actividad mejor considerada, señalada por casi el $50 \%$ de la muestra. Sin embargo, vemos que existe un desajuste entre lo que piensan y lo que hacen, ya que hablar no era una de las actividades que realizaran con más frecuencia.

\subsection{Importancia de estas actividades}

Dentro de su percepción sobre la educación informal, nos interesaba saber qué importancia le otorgaban a esta. Creamos, así, una pregunta con respuesta politómica para que valoraran su importancia respecto a las clases o educación formal. El 75,3\% de los informantes consideró este tipo de educación igual de importante que aquella impartida por un docente en el aula. Por otro lado, 17,9\% la valoró como más importante, mientras que tan solo el $6,8 \%$ pensó que era menos importante que la enseñanza formal. Observemos cómo se distribuyen las respuestas en la siguiente tabla de contingencia:

\begin{tabular}{|c|c|c|c|c|c|}
\hline & \multicolumn{3}{|c|}{ Importancia } & \multirow[b]{2}{*}{ Tota } \\
\hline & & $\begin{array}{c}\text { Más } \\
\text { importante }\end{array}$ & $\begin{array}{c}\text { Menos } \\
\text { importante }\end{array}$ & $\begin{array}{l}\text { Igual de } \\
\text { importante }\end{array}$ & \\
\hline \multirow{2}{*}{$\begin{array}{l}\text { Estancia en } \\
\text { el extranjero }\end{array}$} & No & 19 & 12 & 106 & 137 \\
\hline & Sí & 15 & 1 & 37 & 53 \\
\hline & tal & 34 & 13 & 143 & 190 \\
\hline
\end{tabular}

Quizás la diferencia fundamental que puede observarse en la tabla es que de los 13 estudiantes que otorgan una menor importancia a este tipo de aprendizaje, 12 no han estudiado en el extranjero.

Tras someter estas variables a la prueba de la ji al cuadrado, esta arrojó un valor $p$ de 0,025 ( $p<0,05)$, por lo que podemos considerarlas variables dependientes. Es decir, el nivel de lengua incide en la importancia otorgada a la educación informal.

\subsection{Causas de la poca dedicación a estas actividades}

El último punto del cuestionario pretendía aproximarse a las causas por las que no dedicaban más tiempo a este tipo de aprendizaje. Con este objetivo les ofrecimos tres respuestas ${ }^{19}$ para elegir una, más la posibilidad de escribir otra razón en el caso de que su causa no se correspondiera con aquellas expuestas. Veamos la frecuencia y el porcentaje de las respuestas:

19. Estas tres respuestas se basaron en los datos recopilados en la fase de entrevistas. 


\begin{tabular}{|c|c|c|}
\hline & \multicolumn{2}{|c|}{ Causas } \\
\hline & $\mathrm{f}$ & $\%$ \\
\hline No me interesan, me aburro & 21 & 11,1 \\
\hline No tengo tiempo & 67 & 35,3 \\
\hline No sé cómo acceder a las fuentes & 83 & 43,7 \\
\hline Otro & 19 & 10,0 \\
\hline Total & 190 & 100,0 \\
\hline
\end{tabular}

La tabla revela que el mayor porcentaje de los encuestados $(83 / 43,7 \%)$ alega no saber cómo acceder a las fuentes, el 35,3\% aduce que no tiene tiempo para dedicarse a estas actividades con más asiduidad, mientras que el 11,1\% afirma que no le interesa este tipo de aprendizaje porque se aburre. Por último, el 10\% apunta alguna razón diferente a las indicadas. Veamos cómo se distribuyen las respuestas en relación con la variable estancia en el extranjero:

\begin{tabular}{|c|c|c|c|c|c|c|}
\hline & \multicolumn{4}{|c|}{ Causas } & \multirow[b]{2}{*}{ Total } \\
\hline & & $\begin{array}{l}\text { No me } \\
\text { interesan, } \\
\text { me aburro }\end{array}$ & \multirow{2}{*}{$\begin{array}{r}\begin{array}{c}\text { No tengo } \\
\text { tiempo }\end{array} \\
43\end{array}$} & \multirow{2}{*}{$\begin{array}{r}\begin{array}{c}\text { No sé cómo } \\
\text { acceder a } \\
\text { las fuentes }\end{array} \\
63\end{array}$} & \multirow{2}{*}{$\begin{array}{r}\text { Otra } \\
14\end{array}$} & \\
\hline \multirow{2}{*}{$\begin{array}{l}\text { Estancia en } \\
\text { el extranjero }\end{array}$} & No & 17 & & & & 137 \\
\hline & Sí & 4 & 24 & 20 & 5 & 53 \\
\hline & Total & 21 & 67 & 83 & 19 & 190 \\
\hline
\end{tabular}

La prueba de la ji al cuadrado arrojó un valor p de 0,31 ( $p>0,05)$, de modo que no podemos considerar que exista dependencia entre las variables. El nivel de lengua no influye, por tanto, en las razones aducidas por nuestros informantes.

Respecto a aquel $10 \%$ que argumentó otra causa, cabe mencionar que los encuestados señalaron la pereza que les producía hacer trabajo extra más allá de lo exigido por la enseñanza reglada, también señalaron la dificultad que les suponía acercarse a textos y discursos reales en una lengua extranjera.

\section{Conclusiones}

En las páginas previas hemos ofrecido un acercamiento a las prácticas de educación informal empleadas por los estudiantes universitarios de español en Corea del Sur. Como hemos visto, las distintas actividades señaladas no son realizadas con demasiada frecuencia por estos. De hecho, entre el $60 \%$ y el $70 \%$ de la muestra señaló que no realizaba nunca o casi nunca la mayoría de las actividades propuestas. Usar aplicaciones en el móvil, escuchar música y navegar por internet 
fueron las actividades realizadas con mayor frecuencia. Por otro lado, casi el $50 \%$ de los encuestados consideró que la mejor actividad para aprender español era conversar con amigos o conocidos. A este respecto, cabe destacar un desajuste entre la valoración y la práctica, ya que el 65,2\% admitió no hablar en español nunca o casi nunca. Las dificultades para encontrar personas con las que charlar así como la diferencia horaria con los países donde se habla español, para aquellos que tienen amigos en ellos, justifican en gran parte este hecho. A pesar de los datos expuestos, la percepción sobre este tipo de educación es bastante positiva: el 94,2\% consideró útil este tipo de actividades fuera de la enseñanza formal, asimismo el 75,3\% las consideró igual de importantes que aquellas llevadas a cabo en las clases de ELE. Cabe destacar que los estadísticos empleados revelaron que el nivel de español influía tanto en la percepción de utilidad como en la importancia que los informantes otorgaban a este tipo de prácticas. Del mismo modo, se constató que aquellos que tenían mayor nivel de español realizaban tareas informales con mayor frecuencia que aquellos cuya competencia en la lengua era menor.

Por último, la escasa realización de estas actividades encuentra dos razones fundamentales: la falta de tiempo (43\%) y el no saber cómo acceder a las fuentes (63\%). En relación con esta última causa, los profesores debemos hacer autocrítica y aumentar la información que ofrecemos en clase, no solo sobre los beneficios de este tipo de educación sino sobre los espacios físicos o virtuales donde pueden acceder a materiales diversos. La organización de charlas al inicio de cada semestre para ofrecer o refrescar esta información al alumnado podría resultar de gran ayuda, así como la posibilidad de organizar un seminario sobre recursos para el aprendizaje del español fuera del aula. Solo así tendrán información suficiente para aprovechar la gran cantidad de recursos, actualmente disponibles, que pueden facilitar su adquisición y aprendizaje del español.

\section{Referencias bibliográficas}

Ann, B. M. (2012). «Education in the Republic of Korea: National Treasure or National Headache?». Education Week, 31(16): 38-39.

Arnaiz, P. y Peñate, M. (2004). «El papel de la producción oral (output) en el proceso de aprendizaje de una lengua extranjera (LE): el estudio de sus funciones». Porta Linguarum, 1: 37-59.

Arslan, M. (2011). «Second Language Acquisition in the Context of Informal Education». En: A. Akbarov (ed.). Languages for Specific Purposes in Theory and Practice (2011). Cambridge. Cambridge Scholar Publishing: 194-211.

Bermeo, E. (2014). «South Korea's successful education system: lessons and policy implications for Peru». Korean Social Science Journal, 41: 135-151. 
Chen, X. B. (2013). «Tablets for informal language learning; student usage and attitude». Language Learning $\mathcal{E}$ Technology, 17(1):20-36.

Clough, G.; Jones, A. C; McAndrew, P. y Scanlon, E. (2009). «Informal Learning Evidence in Online Communities of Mobile Device Enthusiasts». En: M. Ally (ed.). Mobile Learning: Transforming the Delivery of Education and Training. Canadá. AU Press: 99-112.

Coombs, P. H., Prosser, R. C. y Ahmed, M. (1973). New Paths to Learning for Rural Children and Youth. Nueva York: International Council for Educational Development.

Corpas, M. D. (2016). «La educación informal en el aprendizaje del inglés como lengua extranjera». Tejuelo, 24:68-91.

Cuadrado, T. (2008). La enseñanza que no se ve. Educación informal en el siglo XXI. Madrid: Narcea Ediciones.

De la Cruz, F. (2014). «Los estados desarrollistas en el este asiáticoः aportaciones institucionales y límites históricos». Revista Iberoamericana de Estudios de Desarrollo, 3(2): 26-49.

Doy le, M. E. (2001). «On being an educator». En* L. D. Richardson y M. Wolf, (eds.). Principles and Practice of Informal Education. Learning through life. Londres-Nueva York. Routledge: 4-16.

Ellinger, T. R. y Carlson, D. L. (1990). «Education in Korea Doing Well and Feeling Bad». Foreing Service Journal, 67(6): 16-18.

Ellis, J. W. (1990), «Informal Education - a Christian Perspective». En: T. Jeffs y M. Smith, (eds.). Using Informal Education. Buckingham: Open University Press.

Farmahini, M.; Hasan, M. y Noroozi, N. (2014). «A Study on Features of Informal Education in Postmodernism». Procedia-Social and Behavioral Sciences, 136 :539-563.

Godwin-Jones, R. (2011). «Emerging technologiesः mobile apps for language learning». Language Learning $\mathcal{E}$ Technology, 15(2): 2-11.

Hernández, V+ (2015). Estudio sobre el Idioma Español en la República de Corea. Realidad y Retos. Seúl: Embajada de España en Corea.

Hultberg, P. et al. (2017). «Education policy in South Korea: A contemporary model of human capital accumulation?». Cogent Economics E Finance 5: 1-16. Jiménez, J. y Cabrera, J. (2011). «El español en Corea del Sur». Enः El español en el mundo. Anuario del Instituto Cervantes 2010-2011. Centro de Investigación y Documentación del Instituto Cervantes (CIDIC): 1-32.

Kvale, S. (2011). Las entrevistas en Investigación Cualitativa. Madrid: Ediciones Morata. 
Lee, J. K. (2006). «Educational Fever and South Korean Higher Education». REDIE, 8(1): 1-14.

Lee, S., Hong, J+y Espelage, D. (2010). «An ecological understanding of youth suicide in South Korea». School Psychology International, 31(5):531-546.

Livingstone., D. W. (2001). Adults' Informal Learning: Definitions, Findings, Gaps and Future Research. NALL Working Paper 21. Toronto: Ontario Institute for Studies in Education.

Mahoney, J. (2001). «What is informal education?». En* L. D. Richardson y M. Wolf (eds.). Principles and Practice of Informal Education. Learning through life. Londres-Nueva York. Routledge: 17-33.

Miró, O. y Álvarez, A. B. (2015). «El perfil del profesor nativo de ELE en Corea del Sur». MarcoELE, 20: 1-30.

Onieva, J. L. (2015). «La enseñanza del español en las universidades de Corea del Sur: handicaps y propuestas didácticas para Hankuk University of foreign studies (Seúl)». Tejuelo, 21: 101-116.

Palacios, I. M. (2006). "Aprendiendo a aprender en el aula de lenguas extranjeras. Las estrategias de aprendizaje y su tratamiento en el aula». En: D. Cassany (dir.). El portfolio europeo de las lenguas y sus aplicaciones en el aula. Madrid. Ministerio de Educación y Ciencia - Secretaría General de Educación: 129-170.

Pole, K. (2009). «Diseño de metodologías mixtas. Una revisión de las estrategias para combinar metodologías cuantitativas y cualitativas». Renglones, 60 * 37 42.

Quintana, J. M. (1991). «La educación más allá de la escuela». En: V. García $\mathrm{Hoz}$ (ed.). Iniciativas sociales en educación informal. Madrid. Ediciones Rialp: 15-61.

RomeA, C. (2011). «Los nuevos paradigmas para los procesos de enseñaza/ aprendizaje en la sociedad del conocimiento en E/LE». Magriberia, 4: 105 116.

Schugurensky, D. (2000), The Forms of Informal Learning: Towards a Conceptualization of the Field, NALL Working Paper 19. Toronto: Ontario Institute for Studies in Education.

Scribner, S. y Cole, M. (1973). «Cognitive Consequences of Formal and Informal Education». Science, 182(4112):553-559.

Setr, M. (2002). Education Fever: Society, Politics, and the Pursuit of Schooling in South Korea. Honolulu: University of Hawaii Press.

Shin, S. y Кон, M. S. (2005). «Korean Education in Cultural Context». Essays in Education, $14: 1-10$. 
Smith, M. K. (2001). «Place, space and informal education». En: L. D. Richardson y M. Wolf (eds.). Principles and Practice of Informal Education. Learning through life. Londres-Nueva York. Routledge: 138-147.

SwaIN, M. (1985). «Communicative competence: some roles of comprehensible input and comprehensible output in its development». En S. Gass y C. G. Madden (eds.). Input and Second Language Acquisition. Rowley, MA. Newbury House: 235-253.

Swain M. (1995). «Three functions of output in second language learning». En: G. Cook y B. Seidlhofer (eds.). Principle and Practice in Applied Linguistics. Oxford. Oxford University Press: 125-144.

Swain, M. y Lapkin, S. (1995). «Problems in output and the cognitive processes they generate: a step towards second language learning». Applied Linguistics, 16(3): 371-391.

Touriñán, J. M. (1996). "Análisis conceptual de los procesos educativos "formales","no formales" e "informales"». Teoría de la educación, 8: 55-79.

Tudor, S. L. (2013)+ «Formal - Non Formal - Informal in Education». ProcediaSocial and Behavioral Sciences, 76: 821-826.

Trilla, J. (1986). «La educación no formal». En: M. Morales (comp.). Educación no formal: lugar de conocimientos. Selección de textos. Primera edición de 2013. Uruguay. Ministerio de Educación y Cultura: 27-50

Wolfe, M. y Richardson, L. D. (2001). «Introduction». En: L. D. Richardson y M. Wolf (eds.). Principles and Practice of Informal Education. Learning through life. Londres-Nueva York. Routledge: xi-xiv. 